\title{
Class I and class II major histocompatibility complex antigens on hepatocytes: Importance of the method of detection and expression in histologically normal and diseased livers
}

\author{
G Senaldi, A Lobo-Yeo, A P Mowat, G Mieli-Vergani, D Vergani
}

\begin{abstract}
Methodological differences in major histocompatibility complex (MHC) antigen detection were investigated on isolated, viable hepatocytes and cryostat hepatic sections from 27 children with liver disorders, six of whom had normal histology. Class I antigens were constantly found on sections using a three step immunoperoxidase technique after acetone/chloroform fixation, other techniques being less sensitive, or on isolated hepatocytes by indirect immunofluorescence alone. With mechanical isolation the percentage of positivity ranged from 85 to $100 \%$, while with collagenase isolation it ranged from 22 to $49 \%$ on immediate testing, and from 53 to $80 \%$ after 24 hour incubation. Class II antigens were only detected in one patient with autoimmune chronic active hepatitis and two with primary sclerosing cholangitis. Flow cytofluorimetric analysis in 11 cases confirmed class II or class I positivity, or both, on isolated hepatocytes, allowing MHC antigen expression on hepatocytes to be measured.

Class I and II antigen detection on hepatocytes is influenced by the technique used. Although class $I$ antigens are invariably expressed on hepatocytes, class II antigens are only found on hepatocytes from patients with immune mediated liver disorders.
\end{abstract}

Class I (HLA-A, -B, -C) and class II (HLADR) antigens are products of the major histocompatibility complex (MHC): they have a central role in the induction and control of the immune response. ${ }^{1}$

Class I antigens are reported to be present on all nucleated cells while class II antigens only occur on limited cell populations, such as monocytes and macrophages, $B$ cells, and activated $T$ cells. ${ }^{2-4}$ Recent studies, however, have challenged these notions. Class I antigens have been shown to have a more restricted distribution than originally postulated, not being observed on corneal endothelium, epithelial cells of exocrine pancreas, and endocrine and nervous cells. They are also expressed with variable intensity in different tissues. ${ }^{56}$ Conversely, it has been shown that class II antigens are more widely expressed in tissues than was previously thought, being found on endothelial cells and a variety of epithelial cells. ${ }^{78}$ In some disorders de novo class II antigen expression might enable cells, otherwise not normally involved in the immune response, to perform antigen presenting cell functions. ${ }^{9-12}$

There are conflicting data on the expression of class I antigens on normal human hepatocytes; some investigators have been unable to detect these molecules, ${ }^{513-15}$ while others have, albeit at low density. ${ }^{616-19}$ There is, however, consensus about the lack of class II antigens on normal hepatocytes. ${ }^{7814151718}$ The expression of class I and II antigens on hepatocytes from patients with several liver diseases, particularly those considered to have an immune component to their pathogenesis, has been intensively investi-

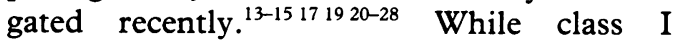
antigens have been consistently detected, especially in areas of liver damage such as piecemeal necrosis and spotty necrosis, the degree of class II antigen expression is disputed. ${ }^{1415242628}$ The published results are not strictly comparable, because they have been obtained with different techniques. Major differences in tissue manipulation, choice of reagents, fixation procedures and staining protocols are evident in these studies. This raises the question of the degree to which results regarding the expression of $\mathrm{MHC}$ antigens on hepatocytes are influenced by the methods chosen.

To address this problem we investigated six histologically normal and 21 diseased liver biopsy specimens from children using the most widely applied methods to detect class I and II antigens. We compared the results obtained using these techniques with those obtained by immunofluorescence staining of viable hepatocytes that had been freshly isolated from the same biopsy specimens.

\section{Methods}

Percutaneous liver biopsy specimens were obtained from 24 children (14 males and 10 females, median age 2 years, range 2 months16 years). The specimens were taken with a $1.4 \mathrm{~mm}$ diameter Menghini needle. Only material not required for purposes of clinical management was used in this study and consisted of 5-10 mm long liver cylinders. Biopsy 
specimens were taken from four patients with no histological abnormalities (two with Criggler-Najjar's syndrome, one with ornithine transcarbamylase deficiency, and one four months after recovery from Reye's syndrome); four with $\alpha$-1-antitrypsin deficiency (protease inhibitor ZZ phenotype); four with extrahepatic biliary atresia; three with idiopathic neonatal hepatitis; two with primary sclerosing cholangitis (PSC); two after liver transplantation (one with acute rejection and one with acute cholangitis); and one with each of the following: $\mathrm{HBsAg}$ and $\mathrm{HBeAg}$ positive chronic persistent hepatitis; $\mathrm{HBsAg}$ positive, $\mathrm{HBeAg}$ negative cirrhosis with minimal hepatitis; autoimmune chronic active hepatitis ( $\mathrm{CAH}$ ); Alagille's syndrome; and glycogen storage disease type IX. Surgical biopsy specimens were obtained from two children, both female, aged 6 and 7 months. One was undergoing surgery for hepatoblastoma and her biopsy specimen comprised a piece of histologically normal liver surrounding the resected lesion. The other was undergoing the Kasai operation as treatment for extrahepatic biliary atresia. Liver tissue was also obtained from a 15 year old organ donor whose liver could not be used for transplantation because the recipient had died during surgery.

The biopsy specimens were divided into equal pieces immediately after collection. Two pieces were recovered with the purpose of comparing the methods for the detection of MHC antigens on isolated hepatocytes with those for hepatocytes in liver sections. One was immersed in RPMI 1640 medium (Gibco Europe, Uxbridge, England) containing 10\% fetal calf serum (FCS) (Gibco Europe) and $20 \mathrm{mmol} / 1$ of EDTA; the other was embedded in OCT medium (BDH, Poole, Dorset) and stored in liquid nitrogen until use. To compare MHC antigen expression on the mechanically isolated hepatocytes with that on hepatocytes isolated enzymatically, a third piece was cut from five biopsy specimens and placed in RPMI containing 10\% FCS and $0.01 \%$ collagenase type IV (Sigma UK).

\section{IMMUNOLOGICAL REAGENTS}

Three murine IgG2a monoclonal antibodies were used: W6/32 directed against a monomorphic determinant on class I antigens ${ }^{29}$; L243 directed against a monomorphic determinant of class II antigens ${ }^{30}$; and BE3F9 directed against bovine insulin as control antibody. ${ }^{31}$ These antibodies were recovered from the supernatant of cultured hybridomas, obtained from the American Type Culture Collection (Rockville, Maryland, USA) and grown for one to two weeks in RPMI with $2 \%$ FCS at $37^{\circ} \mathrm{C}$ in a $5 \%$ carbon dioxide atmosphere. The antibodies were purified by protein A affinity chromatography and stored in phosphate buffered saline (PBS) ( $\mathrm{pH} \mathrm{7 \cdot 2)}$ containing $0.5 \%$ bovine serum albumin and $0.01 \%$ sodium azide at an approximate concentration of $100 \mu \mathrm{g} / \mathrm{ml}$.

Fluorescein isothiocyanate (FITC)- conjugated rabbit anti-mouse immunoglobulin $\mathrm{F}\left(\mathrm{ab}^{\prime}\right)_{2}$ fragment, peroxidase-conjugated rabbit anti-mouse immunoglobulin antiserum, and peroxidase-conjugated swine antirabbit immunoglobulin antiserum were the antisera used (all purchased from Dakopatts, High Wycombe, England).

Immunological reagents were used at saturating concentrations determined in preliminary titration experiments.

DETECTION OF CLASS I AND II ANTIGENS ON LIVER SECTIONS

Thirty six serial $5 \mu \mathrm{m}$ thick sections were cut from all frozen liver samples in an OTF/AS cryostat (Bright, Runcorn, England) on to polylysine coated slides and air dried for three to six hours. Twelve combinations of different fixation and staining procedures were then used.

The 36 sections were divided into four groups of nine: nine were fixed by immersion in a 1 in 1 acetone/chloroform mixture for five minutes ${ }^{2124}$; nine were fixed in acetone for 10 minutes ${ }^{6131425}$; nine were fixed in ethanol for 15 minutes $^{28}$; nine were left unfixed. All the sections were washed extensively in PBS, treated with $0.3 \% \mathrm{H}_{2} \mathrm{O}_{2}$ in PBS for 20 minutes to block endogenous peroxidase, washed again and incubated with PBS containing $10 \%$ normal rabbit serum and $10 \%$ normal swine serum to block sites of non-specific binding.

The nine sections within each group were then assembled in three sets of three, and each section in a set was incubated for 30 minutes with either anti-class I, anti-class II, or control monoclonal antibody, diluted 1 in 40,1 in 80 , and 1 in 40 , respectively, to achieve optimal staining. After washing for $15 \mathrm{~min}$ utes in three changes of PBS one set within each group was treated with FITC-conjugated rabbit anti-mouse immunoglobulin $\mathrm{F}\left(\mathrm{ab}^{\prime}\right)_{2}$ fragment and the other two with peroxidase-conjugated rabbit anti-mouse immunoglobulin, both diluted 1 in 20 in PBS containing $5 \%$ normal human $\mathrm{AB}$ serum to block cross reactivity with human immunoglobulin. After washing as above the sections stained for indirect immunofluorescence were mounted in $90 \%$ glycerol in PBS and examined under ultraviolet microscopy. One of the two remaining sets of the peroxidase reaction was developed by the addition of PBS containing diaminobenzidine $(0.6 \mathrm{mg} / \mathrm{ml})$ and $\mathrm{H}_{2} \mathrm{O}_{2}(0.03 \%)$ according to a standard procedure, rinsed in tap water, counterstained with Mayer's haematoxylin, mounted in glycerol/PBS and examined under light microscopy. The remaining set was further incubated with peroxidase-conjugated swine anti-rabbit immunoglobulin diluted 1 in 20 in PBS containing 5\% normal human AB serum and the peroxidase reaction was developed as above.

Expression of MHC antigens on hepatocytes was graded by a single observer unaware of clinical data using a semiquantitative scale as follows: $0=$ negative; 
$1=$ minimal $; \quad 2$ = weak; 3 = moderate; $4=$ intense. Expression of class I and II antigens on Kupffer and sinusoidal cells served as a reference for evaluating the staining of the preparations.

DETECTION OF CLASS I AND II ANTIGENS ON ISOLATED HEPATOCYTES

Isolation of hepatocytes

Isolated, viable hepatocytes were prepared by mechanical or enzymatic dispersion of the liver biopsy specimens, as previously described. ${ }^{32}$

For the mechanical isolation of hepatocytes, the portion of biopsy specimen collected in RPMI supplemented with EDTA was teased apart using two 19 gauge needles in a small Petri dish. The disrupted tissue was suspended in RPMI and filtered through nylon wool ("Leuko-Pak", Travenol, Thetford, England) to eliminate large clumps of cells and debris. The filtrate was then centrifuged at $100 \mathrm{~g}$ for five minutes, the pellet resuspended in a drop of RPMI, and the hepatocytes counted. Hepatocytes could be distinguished from other cellular components by virtue of their characteristic cytological features: they are large and often polygonal and possess a small nucleolous, which is frequently duplicated, and abundant granular cytoplasm. The cell yield ranged from 2 to $6 \times 10^{4}$ hepatocytes. Hepatocyte viability was consistently between $60 \%$ and $80 \%$, as assessed using Trypanblue. Hepatocytes thus prepared were tested immediately.

For the enzymatic isolation of hepatocytes, the portion of biopsy specimen collected in RPMI containing collagenase was incubated in the same medium for three hours at $37^{\circ} \mathrm{C}$ under constant agitation. The disrupted tissue was filtered and washed and the hepatocyte number and viability assessed as described above. Values were similar to those obtained after mechanical isolation. About half of the hepatocytes yielded by enzymatic disruption were tested immediately; the remainder were incubated in RPMI with $10 \%$ FCS for 24 hours at $37^{\circ} \mathrm{C}$ in a $5 \%$ carbon dioxide atmosphere and stained.

\section{Microscopic analysis}

Isolated hepatocytes were stained in suspension using a two step indirect immunofluorescence technique. Hepatocytes were adjusted to a final concentration of $5-15 \times 10^{3}$ cells in $100 \mu \mathrm{l}$ of RPMI. Three such preparations were obtained from each disruption experiment and also from the hepatocytes incubated for 24 hours after enzymatic disruption. The hepatocyte suspension was incubated with $5 \mu$ l of anti-class I, anti-class II, or control monoclonal antibody for 30 minutes at $4^{\circ} \mathrm{C}$, washed twice by centrifugation in RPMI at $100 \times g$, resuspended in about $100 \mu \mathrm{l}$ of medium and incubated with $5 \mu$ l of FITC-conjugated rabbit anti-mouse immunoglobulin $\mathrm{F}\left(\mathrm{ab}^{\prime}\right)_{2}$ fragment for 30 minutes at $4^{\circ} \mathrm{C}$. After washing, the cells were resuspended in a drop of RPMI and examined using an ultraviolet microscope (Polyvar, Reichert-Jung, Vienna, Austria).

In five cases, where sufficient biopsy material was available, mechanically isolated hepatocytes were also stained as a cytospin preparation using a two step indirect immunoperoxidase technique. Hepatocytes, resuspended as above in $100 \mu \mathrm{l}$ of RPMI, were centrifuged (Shandon-Elliott, Runcorn, England) at $100 \times g$ for three minutes on a slide precoated with $0.01 \%$ poly-l-lysine (Sigma) in distilled water. A cytospin for each of the three monoclonal antibodies was prepared, air dried for one hour, fixed in acetone for 10 minutes, and then processed as described for immunoperoxidase staining of liver sections.

\section{FLOW CYTOFLUORIMETRIC ANALYSIS}

In a preliminary experiment cells isolated from the liver of the organ donor were analysed by flow cytofluorimetry using a Facstar (Becton Dickinson, Oxford, England). The standard three parameter protocol of forward angle and wide angle light scattering and green fluorescence was used. A discrete cell population was identified, sorted, and analysed (fig 1A). To assess its purity, a cytospin preparation of this population was stained with haematoxylin and eosin and shown to be composed exclusively of hepatocytes (fig 1B).

Flow cytofluorimetric analysis was then used in 11 cases in which adequate numbers of indirect immunofluorescence stained hepatocytes had remained after examination

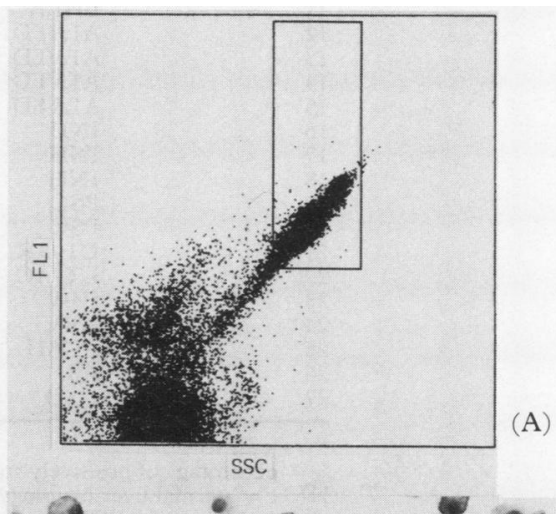
mechanically disrupted liver biopsy specimen analysed in a flow cytofluorimeter for light scattering (horizontal axis, SSC) and autofiuorescence (vertical axis, FL1). The cells within the rectangle (panel $A$ ) were sorted and all shown to be hepatocytes in a cytospin preparation (panel $B$, haematoxylin and eosin). 
by ultraviolet microscopy. Distribution of fluorescence intensity of the hepatocyte populations was analysed and the median fluorescence intensity calculated and expressed in log transformed arbitrary units using the Consort-30 software program (Becton Dickinson) on a computer unit (Hewlett-Packard, Wokingham, England) connected with the flow cytofluorimeter.

\section{Results}

EXPRESSION OF CLASS I AND II ANTIGENS ON LIVER SECTIONS

Tables 1 and 2 summarise the results for the expression of class I and II antigens on liver sections, as detected using different combinations of fixation and staining procedures. Hepatocytes expressing class I antigens were found in all the patients studied only when the three step immunoperoxidase technique after fixation in acetone/chloroform was used. Considerable variations in the sensitivity of class I detection were observed between the indirect immunofluorescence and immunoperoxidase methods, and among the different fixation procedures. Independently of the type of fixation, immunoperoxidase staining proved more sensitive than indirect immunofluorescence, permitting both the detection of a higher number of positive patients and higher scores of staining intensity. When immunoperoxidase staining was performed in three steps, higher sensitivities were obtained, and in two patients with histologically normal liver, one of whom was the organ donor, only the application of three step immunoperoxidase after fixation in acetone/chloroform allowed class I antigens on hepatocytes in liver sections to be detected (figs $2 \mathrm{~A}-\mathrm{C})$. On unfixed liver sections, stained by indirect immunofluorescence, two step immunoperoxidase or three step immunoperoxidase, class I antigens were identified in only a minority of the cases which gave positive results on isolated hepatocytes. The acetone/ chloroform mixture proved better than ethanol

Table 1 Expression of class I and II antigens on liver cell membrane, as detected either on isolated hepatocytes or on liver sections

\begin{tabular}{|c|c|c|c|c|c|}
\hline \multirow[b]{2}{*}{ Case No } & \multirow[b]{2}{*}{ Diagnosis } & \multicolumn{2}{|c|}{ Liver sections ${ }^{\star}$} & \multicolumn{2}{|c|}{ Isolated hepatocytes $\dagger$} \\
\hline & & Class I & Class II & Class I & Class II \\
\hline 1 & $\mathrm{NLH}$ & 12 & 0 & 94 & 0 \\
\hline 2 & $\mathrm{NLH}$ & 10 & 0 & 97 & 0 \\
\hline 3 & $\mathrm{NLH}$ & 3 & 0 & 91 & 0 \\
\hline 4 & $\mathrm{NLH}$ & 1 & 0 & 70 & 0 \\
\hline 5 & NLH & 16 & 0 & 96 & 0 \\
\hline 6 & NLH & 2 & 0 & 58 & 0 \\
\hline 7 & EHBA & 24 & 0 & 98 & 0 \\
\hline 8 & EHBA & 12 & 0 & 100 & 0 \\
\hline 9 & EHBA & 11 & 0 & 100 & 0 \\
\hline 10 & EHBA & 14 & 0 & 100 & 0 \\
\hline 11 & EHBA & 9 & 0 & 95 & 0 \\
\hline 12 & A1ATD & 20 & 0 & 100 & 0 \\
\hline 13 & AlATD & 10 & 0 & 70 & 0 \\
\hline 14 & A1ATD & 11 & 0 & 98 & 0 \\
\hline 15 & AlATD & 8 & 0 & 90 & 0 \\
\hline 16 & INH & 17 & 0 & 97 & 0 \\
\hline 17 & INH & 12 & 0 & 100 & 0 \\
\hline 18 & INH & 10 & 0 & 99 & 0 \\
\hline 19 & PSC & 24 & 16 & 100 & 20 \\
\hline 20 & PSC & 22 & 0 & 100 & 10 \\
\hline 21 & LT-AR & 17 & 0 & 100 & 0 \\
\hline 22 & LT-AC & 10 & 0 & 70 & 0 \\
\hline 23 & $\mathrm{HBV}$ & 3 & 0 & 50 & 0 \\
\hline 24 & HBV & 8 & 0 & 56 & 0 \\
\hline 25 & ACAH & 29 & 27 & 100 & 90 \\
\hline 26 & AS & 26 & 0 & 99 & 0 \\
\hline 27 & GSD & 2 & 0 & 96 & 0 \\
\hline
\end{tabular}

$\star=$ total score.

$+=$ percentage of positively stained hepatocytes.

$\mathrm{NLH}=$ normal liver histology; A1ATD $=\alpha-1$-anti-trypsin deficiency; EHBA $=$ extrahepatic biliary atresia; INH $=$ idiopathic neonatal hepatitis; PSC = primary sclerosing cholangitis; LT-AR = liver transplantation complicated by acute rejection; LT$\mathrm{AC}=$ liver transplantation complicated by acute cholangitis; $\mathrm{HBV}=$ chronic hepatitis $\mathrm{B}$ virus infection; $\mathrm{ACAH}=$ autoimmune chronic active hepatitis; $A S$ = Alagille's syndrome; GSD = glycogen storage disease type IX.

Table 2 Expression of class I and II antigens on liver sections as detected by different combination of fixation procedures and staining techniques

\begin{tabular}{|c|c|c|c|c|c|}
\hline \multirow[b]{2}{*}{ Staining technique } & \multirow[b]{2}{*}{ Fixation procedure } & \multicolumn{2}{|l|}{ Class I } & \multicolumn{2}{|l|}{ Class II } \\
\hline & & $\begin{array}{l}\text { Number of cases } \\
\text { positive } 27\end{array}$ & Score & $\begin{array}{l}\text { Number of cases } \\
\text { positive/ } 27\end{array}$ & Score \\
\hline $\begin{array}{l}\text { Indirect } \\
\text { immunofluorescence } \\
\text { Two step immunoperoxidase } \\
\text { Three step } \\
\text { immunoperoxidase }\end{array}$ & 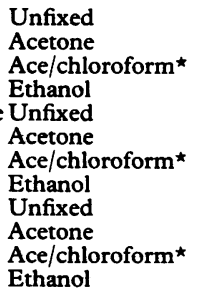 & $\begin{array}{l}5 \\
12 \\
17 \\
12 \\
11 \\
17 \\
25 \\
17 \\
11 \\
18 \\
27 \\
18\end{array}$ & $\begin{array}{r}6 \\
15 \\
22 \\
16 \\
12 \\
30 \\
58 \\
32 \\
13 \\
34 \\
65 \\
40\end{array}$ & $\begin{array}{l}2 \\
2 \\
2 \\
2 \\
2 \\
2 \\
2 \\
2 \\
2 \\
2 \\
2 \\
2\end{array}$ & $\begin{array}{l}2 \\
3 \\
4 \\
3 \\
3 \\
4 \\
5 \\
4 \\
3 \\
4 \\
4 \\
4\end{array}$ \\
\hline
\end{tabular}

Score = total score grading expression of MHC antigens on hepatocytes, formulated as described in the text.

$\star 1 / 1$ mixture of acetone and chloroform. 
Figure 2 Class I antigen expression in liver sections from a patient with no histological abnormalities (previous Reye's syndrome). The sections were fixed in acetone and chloroform. Nonparenchymal cells are positively stained. No hepatocyte staining is visible on the sections treated by either indirect immunofluorescence (panel A) or two step immunoperoxidase (panel B). A weak liver cell membrane staining is present on the section processed by three step immunoperoxidase (panel C).

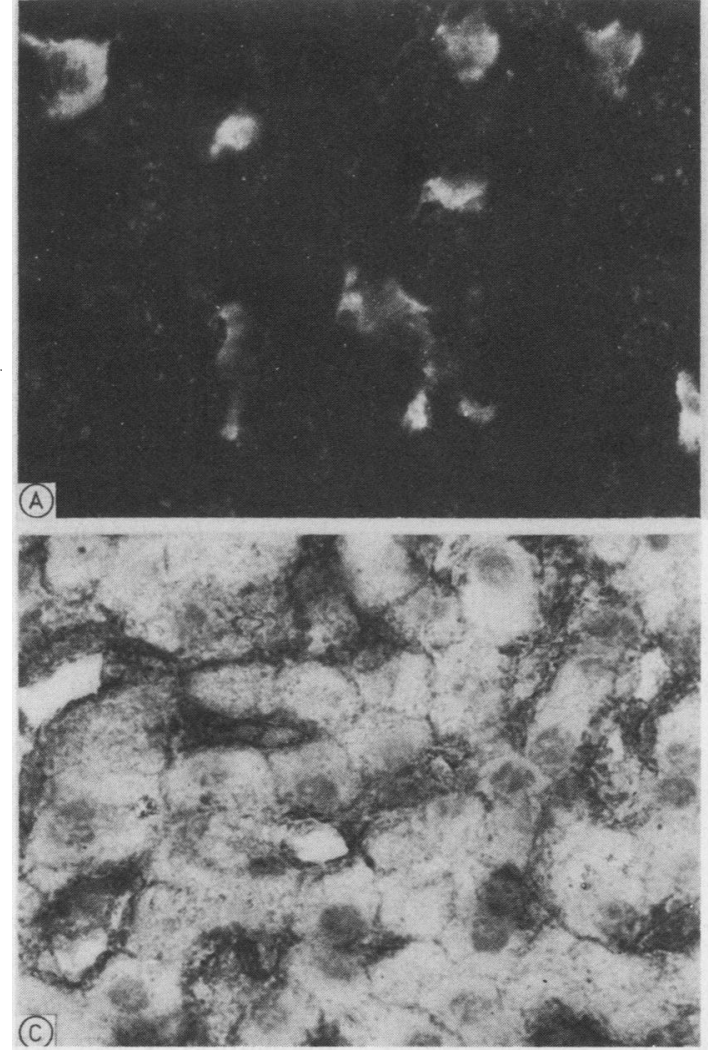

and acetone alone, not only providing greater sensitivity, but also better preservation of the liver tissue. The staining for class I and II antigens using acetone/chloroform fixation of the liver section from a liver transplant recipient with acute rejection is shown in figs 3A-B.

Class II antigens were detected on liver sections from the patient with autoimmune chronic active hepatitis and one of the two patients with primary sclerosing cholangitis, either by indirect immunofluorescence or two or three step immunoperoxidase (fig 4). Class II antigens, however, were not observed on any of the histological preparations from the other patient with primary sclerosing cholangitis, although they had been detected on the isolated hepatocytes.

Major histocompatibility complex antigen expression, whether class I or class II, was always detected both on the membrane and, though much more weakly, in the cytoplasm. Stronger cytoplasmic staining was observed on the liver sections left unfixed; on these sections, however, a high background staining was also found using the irrelevant control monoclonal antibody. According to the number of stained hepatocytes and to the intensity of the staining itself, the pattern of positivity ranged from a faint and scattered form to a strong and diffuse reticular (honeycomb) pattern (fig 5A-B). In autoimmune chronic active hepatitis and primary sclerosing cholangitis expression of class I and II antigens was stronger on the hepatocytes at the periphery of the hepatic lobule than in those occupying a central position (fig 6).

No staining was seen on liver sections when the irrelevant monoclonal antibody was used,

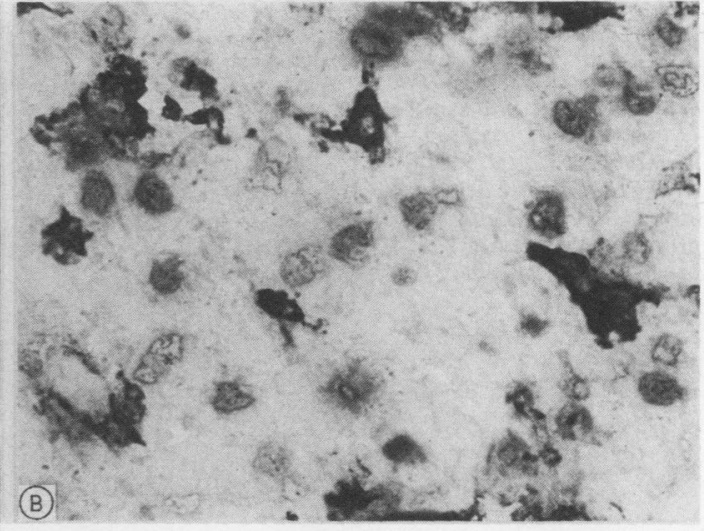

although a weak cytoplasmic staining was observed occasionally on the sections left unfixed.

EXPRESSION OF CLASS I AND II ANTIGENS ON ISOLATED HEPATOCYTES

\section{Ultraviolet.microscopy}

Class I antigens were detected on isolated hepatocytes from all the subjects investigated irrespective of the method of disruption (table 1). In nine cases $100 \%$ of the hepatocytes were positively stained for class I antigens, and in the remaining 18 between $50 \%$ and $99 \%$ of the hepatocytes were positive (median $96 \%$ ). The six histologically normal patients had values between $58 \%$ and $97 \%$ (median $93 \%$ ). In all cases the staining pattern was linear along the

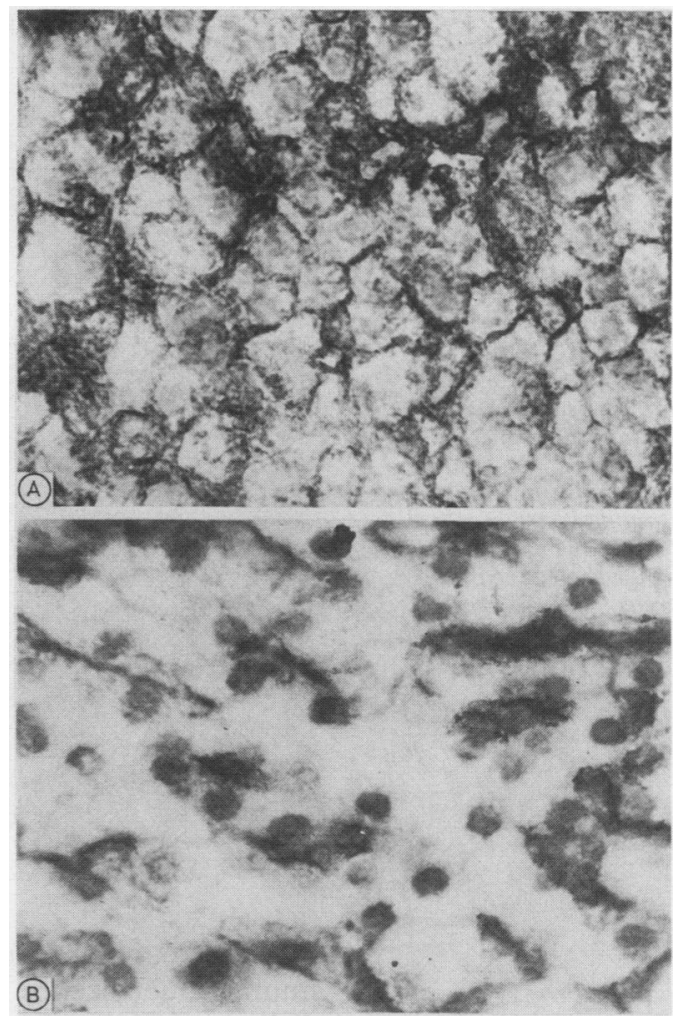

Figure 3 Liver sections from a patient with liver allograft and acute rejection stained for class I antigens (panel $A$ ) and class II antigens (panel $B$ ). The sections were fixed in acetone and chloroform and treated by three step immunoperoxidase. The diffuse positivity for hepatocyte membrane staining observed in panel $A$ contrasts with the negative hepatocyte staining in panel $B$, where only non-parenchymal cells are stained. 
Figure 4 Liver section from a patient with autoimmune chronic active hepatitis stained for class II antigens by three step immunoperoxidase. $A$ membrane staining is present configurating the so-called "honeycomb" pattern.

Figure 5 Liver sections extrahepatic biliary patient with autoimmune chronic active hepatitis (panel B) stained for class I antigens by three step immunoperoxidase. The staining differs in intensity and distribution, being faint and scattered on the first section (panel $A$ ) and strong and diffuse

(honeycomb pattern) on the second (panel $B$ ). strong and diffuse liver cell from a patient with atresia (panel $A$ ) and $a$
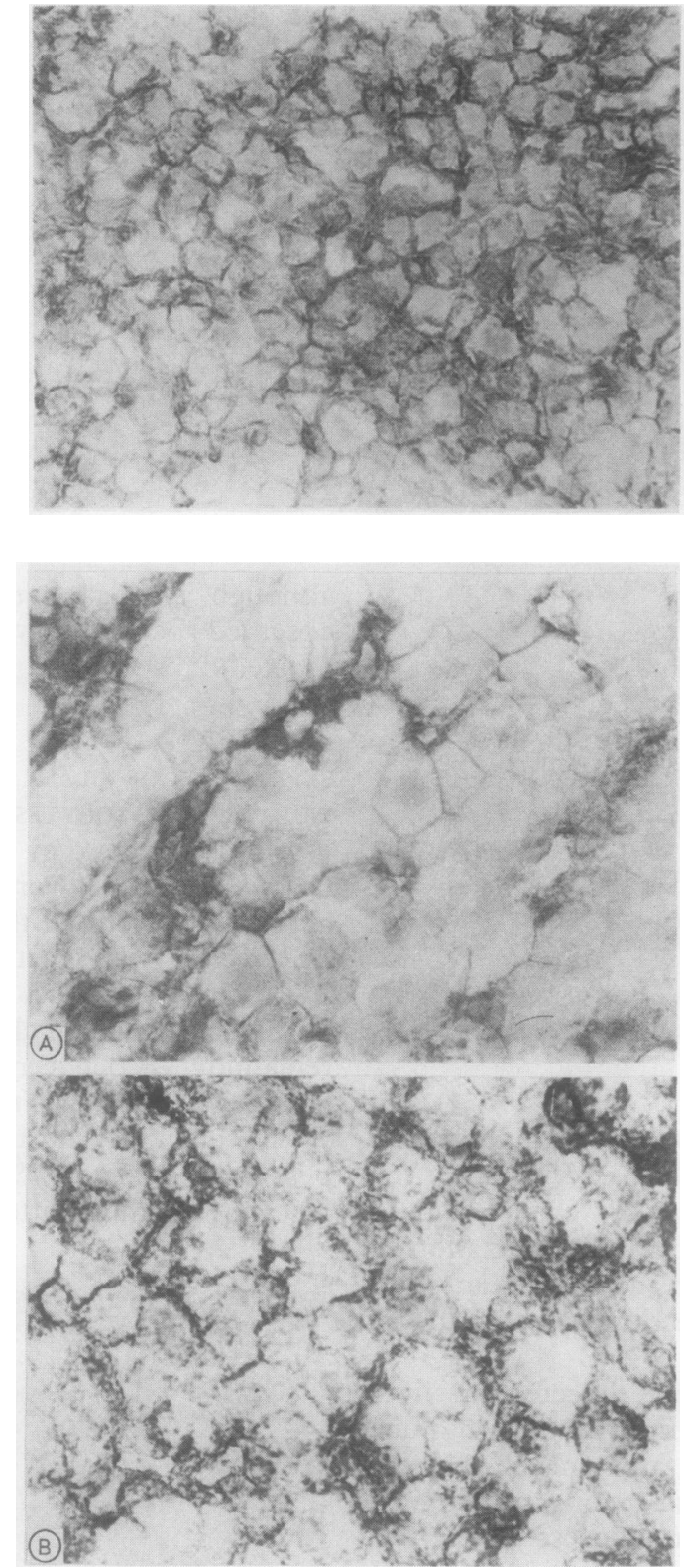

Figure 6 Liver section from a patient with primary sclerosing cholangitis stained for class I antigens. The positivity for hepatocyte membrane staining is stronger at the periphery of the hepatic lobule than in central areas.

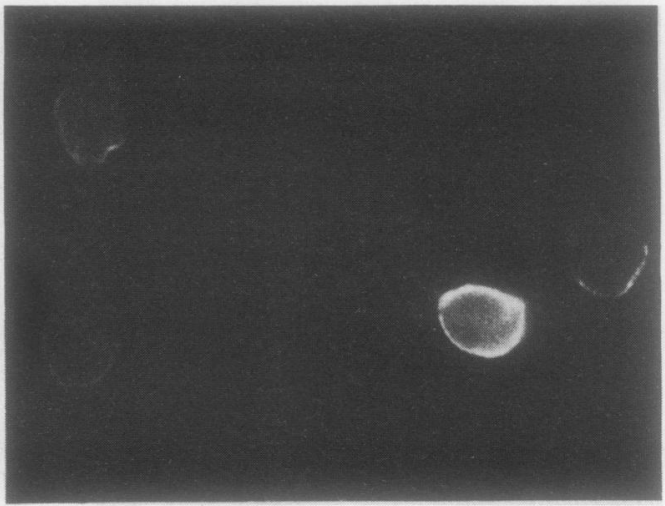

Figure 7 Isolated hepatocytes from a patient with primary sclerosing cholangitis stained by

immunofluorescence for class I antigens. The staining shows a linear pattern on the cell membrane varying in intensity from hepatocyte to hepatocyte.

hepatocyte to hepatocyte (fig 7). In these patients $100 \%$ of the hepatocytes were positive for class I antigens, the fluorescence staining being particularly strong in $10-90 \%$ (median $45 \%$ ) of the cells.

In the five subjects in whom both mechanical and enzymatic disruption were performed, the use of collagenase resulted in a reduction of the percentage of cells expressing class I, which in four cases increased again after 24 hour culture (table 3).

Class II antigens were absent on the isolated hepatocytes from all subjects, apart from those with primary sclerosing cholangitis and autoimmune chronic active hepatitis, in whom $10 \%, 20 \%$, and $90 \%$ of hepatocyte positivity, respectively, were found (table 1 ). The staining had a linear pattern along the hepatocyte membrane (fig 8), as observed for class I antigens, and in the case of autoimmune chronic active hepatitis a strong fluorescence intensity was seen.

No positive hepatocyte staining was detected in any of the patients by using the control monoclonal antibody.

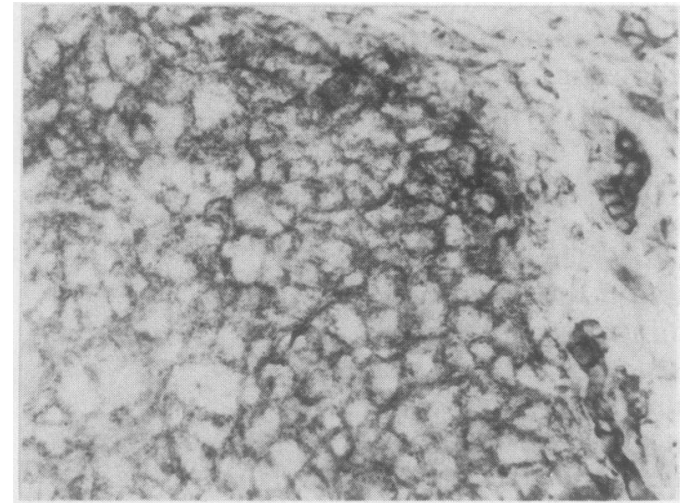

Flow cytofluorimetry

All 11 cases analysed by flow cytofluorimetry were positive for class I antigen expression. Flow cytofluorimetric analysis confirmed the lower fluorescence intensity of enzymatically prepared liver cells when compared with mechanically isolated ones and their increase in fluorescence intensity after 24 hour culture (fig 9).

The child with autoimmune chronic active hepatitis and the only child with primary sclerosing cholangitis tested by flow cytofluorimetry were also positive for class II antigens (fig 10).

Hepatocytes stained using the control mono-

hepatocyte membrane, as assessed by indirect immunofluorescence on isolated hepatocytes. A linear staining pattern was also seen with immunoperoxidase in all five cases from which a cytospin preparation was available. In the patients with primary sclerosing cholangitis, autoimmune chronic active hepatitis, and liver allograft with acute rejection, differences in fluorescence intensity were noticed from

\section{Discussion}

This study shows that the detection of class I and class II antigens on human hepatocytes is 
Table 3 Percentage staining for class I antigens on hepatocytes isolated by mechanical or enzymatical disruption

\begin{tabular}{llll}
\hline & & Enzymatic disruption \\
\cline { 3 - 4 } Patients & $\begin{array}{l}\text { Mechanical } \\
\text { disruption }\end{array}$ & $\begin{array}{l}\text { Immediately after } \\
\text { preparation }\end{array}$ & $\begin{array}{l}\text { After 24 hour culture } \\
\text { in medium }\end{array}$ \\
\hline A & 85 & 22 & 60 \\
B & 100 & 49 & 81 \\
C & 92 & 40 & 70 \\
D & 90 & 42 & 42 \\
E & 95 & 22 & 53 \\
\hline
\end{tabular}

Results are expressed as percentages of positively stained hepatocytes.

Figure 8 Isolated hepatocyte from a patient with primary sclerosing cholangitis stained for class II antigens. The staining shows a linear pattern on the cell membrane (immunofluorescence).
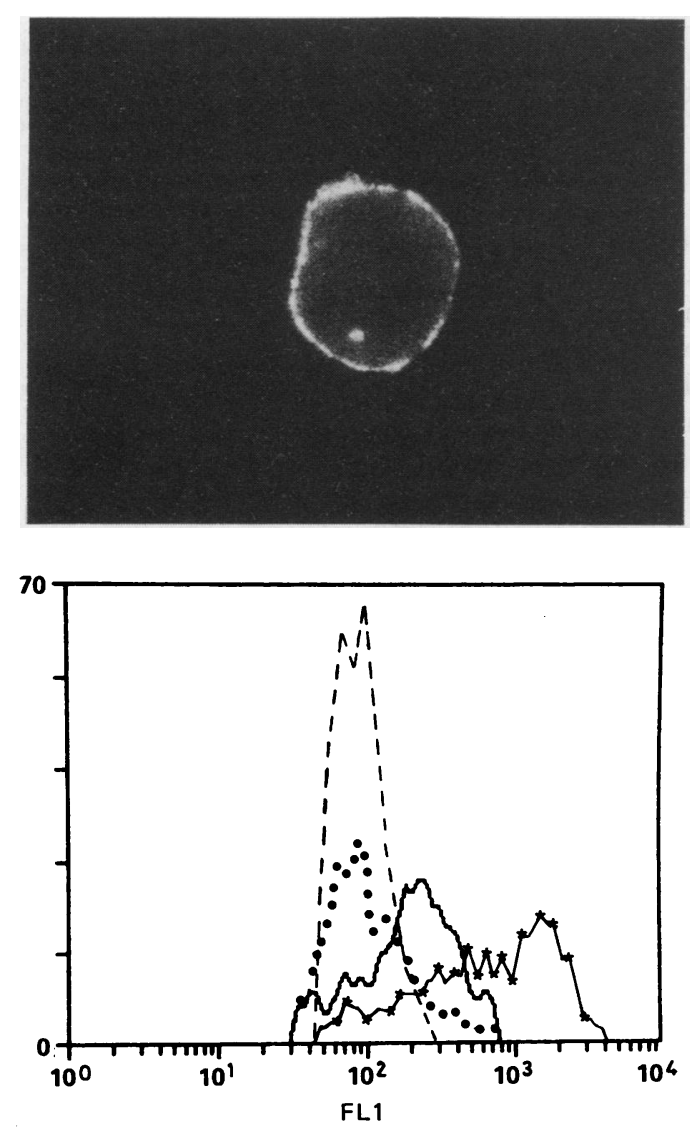

Figure 9 Pattern of fluorescence intensity of the hepatocytes from a patient with extrahepatic biliary atresia, analysed by flow cytofluorimetry. On the vertical axis is represented the cell number in linear scale, on the horizontal axis the fluorescence intensity in logarithmic scale (FL1). Broken line = mechanically isolated hepatocytes stained with control monoclonal antibody (median fluorescence intensity, $M F I=127$ ). Asterix line = mechanically isolated hepatocytes stained for class I antigens ( $M F I=16.2)$. Dots = enzymatically isolated hepatocytes stained for class I antigens before 24 hour culture $(M F I=132)$. Solid line = enzymatically isolated hepatocytes stained for class I antigens after 24 hour culture (MFI = 147).

greatly influenced by the method used, and that the most sensitive results are obtained by staining in suspension mechanically isolated hepatocytes with an indirect immunofluorescence technique. It also shows the feasibility of analysing and quantifying the expression of surface molecules by flow cytofluorimetry, such as $\mathrm{MHC}$ antigens, on a pure population of hepatocytes.

On liver sections, when different fixatives were compared, the mixture of acetone and chloroform provided the best results, giving

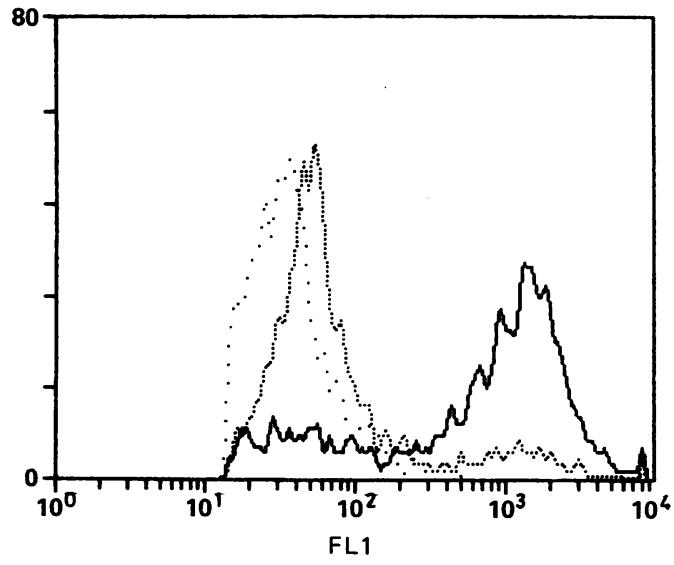

Figure 10 Flow cytofluorimetric pattern of fluorescence intensity of the hepatocytes from a patient with autoimmune chronic active hepatitis stained with irrelevant (widely spaced dotted line), anti-class I (solid line), and anti-class II (closely spaced dotted line) monoclonal antibodies. On the vertical axis is represented the cell number in linear scale, on the horizontal axis the fluorescence intensity in logarithmic scale (FL1).

Hepatocytes display only autofluorescence when stained by an irrelevant monoclonal antibody (median fluorescence intensity, $M F I=114$ ); stronger fluorescence intensity when stained for class II antigens $(M F I=145)$; even stronger fluorescence intensity when stained for class I antigens $(M F I=173)$.

the highest number of positive cases and the strongest staining intensity. Unfixed liver sections proved a poor substrate for staining, possibly because of bad preservation of the liver tissue, so that even the strong expression of class I and II antigens on non-parenchymal cells was difficult to visualise. Furthermore, the background staining was high and genuine membrane staining was hard to detect. It has been suggested that staining for $\mathrm{MHC}$ antigens is greatly decreased in fixed cryostat liver sections, when compared with unfixed sections. ${ }^{26}$ The observation that cytoplasmic staining was seen on unfixed liver sections when an irrelevant control monoclonal antibody was used suggests that, on the contrary, unfixed liver tissue reacts non-specifically with the protein reagents with which it is incubated.

In detecting class I and II antigens on liver sections, immunoperoxidase gave a stronger positive staining, combined with a weaker background, than immunofluorescence, enhancing the detection of the targeted membrane antigens. In one case it was possible to detect class I antigens on hepatocytes in liver sections only when using a three step immunoperoxidase technique, and, overall, the staining intensity was the highest using this technique. Thus the additional sensitivity introduced by the use of a second peroxidaseconjugated reagent may be the determining factor in some cases for the successful detection of MHC antigens. Given the other general advantages of immunoperoxidase over immunofluorescence, such as the permanence of staining and compatibility with counterstaining that permits simultaneous histological analysis, this seems to be the method of choice to investigate presence and distribution of MHC antigens in liver sections.

The staining of freshly isolated, viable hepatocytes in suspension proved the most 
sensitive technique to determine membrane expression of MHC antigen, as evidenced by the case of primary sclerosing cholangitis in which class II antigens were identified by immunofluorescence staining of isolated hepatocytes, but not by any of the other histological techniques used. A further advantage is the relative rapidity of this technique. This method also provides the opportunity for counting the positively stained hepatocytes and of analysing them by flow cytofluorimetry, so that an objective visual representation of the fluorescence intensity range can be derived and the median fluorescence intensity calculated. Class I antigen expression on enzymatically isolated hepatocytes has been previously reported on a small number of biopsy specimens, ${ }^{17}$ although no attempt was made to quantify the positivity. An important new finding of our study is that enzymatically isolated hepatocytes show a reversible decrease in class I expression. The fact that after 24 hour culture the same hepatocytes reacquire class I antigens indicates that hepatocytes have the intrinsic ability to synthetise and express these molecules on their membrane in vitro. The percentage of positive cells remained, however, lower than that obtained by mechanical disruption, suggesting that the action of collagenase may interfere with the preservation of intact molecules, such as MHC antigens, exposed on the hepatocyte surface. This, however, contrasts with previous reports which indicate that membrane integrity may be better preserved on enzymatically than on mechanically isolated hepatocytes. ${ }^{33}$ Such discrepancy probably derives from differences in the technique of mechanical disruption, as suggested by the higher percentage of viable mechanically isolated hepatocytes we obtained when compared with those of previous reports. ${ }^{33}$

When staining mechanically isolated hepatocytes in suspension with an indirect immunofluorescence technique was used to test a series of histologically normal or diseased livers, MHC class I antigens were invariably detected on hepatocytes from both normal and diseased livers, which indicates that these glycoproteins are constitutively expressed on the liver cells. The absence of class I antigens on normal hepatocytes reported by other authors $^{513-15}$ is likely to reflect the low sensitivity of the techniques used rather than representing a genuine biological phenomenon, although an important difference exists between the present study and those reported-that is, our patients are children. We have also shown that unlike class I, class II antigens are not detected on normal hepatocytes, which indicates that they are either absent or expressed at a very low density by liver cells. This agrees with the findings of previous reports..$^{714151718}$ Hepatocytes, however, have the potential to express class II antigens, as they do in immune mediated disorders such as autoimmune chronic active hepatitis and primary sclerosing cholangitis.

We thank Mr D C Davies for performing the flow cytofluorimetric analysis.

Dr Senaldi was supported by The Royal Society, and Drs LoboYeo and Mieli-Vergani by the Children's Liver Disease Foundation, formerly the Michael McGough Foundation against Liver Disease in Children.
1 Benacerraf B. Role of MHC gene products in immune egulation. Science 1981;212:1229-35.

2 Berah $M$, Hors J, Dausset J. A study of HLA antigens in human organs. Transplantation 1970;9:185-92.

3 McKenzie IFC, Morris PJ. Leukocyte antigens in renal transplantation. II. HL-A and ABO antigens on kidney cells in culture. In: Terasaki PI, ed. Histocompatibility testing Copenhagen: Munksgaard, 1970:309-21.

4 Amos DB, Kotsyu DD. HLA: a central immunological agency in man. Adv Hum Genet 1980;10:137-208.

5 Fleming KA, McMichael A, Morton JA, et al. Distribution of HLA class I antigens in normal human tissue and in mammary cancer. J Clin Pathol 1981;34:779-84.

6 Daar S, Fuggle SV, Fabre JW, et al. The detailed distribution of HLA-A, B, C antigens in normal human organs. Transplantation 1984;38:287-92.

7 Natali PG, De Martino C, Quaranta V, et al. Expression of Ia-like antigens in normal non-lymphoid tissue. Transplantation 1981;31:75-8.

8 Daar S, Fuggle SV, Fabre JW, et al. The detailed distribution of MHC class II antigens in normal human organs. Transplantation 1984;38:293-8.

9 Hanafusa T, Pujol Borrel L, Bottazzo GF. Aberrant expression of HLA DR antigen on thyrocytes in Graves' disease: relevance for autoimmunity. Lancet 1983;ii:1111-15.

10 Londei M, Lamb JR, Bottazzo GF, et al. Epithelial cells expressing aberrant MHC class II determinants can present antigen

11 Fontana A, Fiers W, Wekerle H. Astrocytes present myelin basic protein to encephalitogenic $\mathrm{T}$ cell line. Nature 1984;307:273-6.

12 Umetsu DT, Pober JS, Jabara HH, et al. Human dermal fibroblast present tetanus toxoid antigen to antigenspecific T cell clones. J Clin Invest 1985;76:254-60.

13 Barbatis C, Woods J, Morton JA, et al. Immunohistochemical analysis of HLA (A, B, C) antigens in liver disease using a monoclonal antibody Gut 1981;22:985-91.

14 Fukusato T, Gerber MA, Thung SN, et al. Expression of HLA class I antigens on hepatocytes in liver disease. $A m \mathrm{~J}$ Pathol 1986;123:264 70

15 Franco A, Barnaba V, Natali PG, et al. Expression of class I and class II major histocompatibility antigens on human hepatocytes. Hepatology 1988;8:449-54

16 Governa $M$, Biguzzi S. $\beta_{2}$-microglobulin distribution in human normal tissue. Eur J Immunol 1976;6:830-1.

17 Montano L, Miescher G, Goodall AH, et al. Hepatitis B virus and HLA antigen display in the liver during chronic hepatitis B virus infection. Hepatology 1982;2:557-61.

18 Lautenschlager I, Taskinen $\mathrm{E}$, Inkinen $\mathrm{K}$, et al. Distribution of the major histocompatibility complex antigens on different cellular components of human liver. Cell Immunol 1984;85:191-200.

19 Nagafuchi Y, Hobbs KEF, Thomas HC, Scheuer PJ. Expression of $\beta_{2}$-microglobulin on hepatocytes after liver Expression of $\beta_{2}$-microglobulin on hepat

20 De Vos R, De Woolf-Peteers C, Van den Oord JJ, Desmet V. Ultrastructural immunocytochemical demonstration of HLA class I antigens in human pathological liver tissue. Hepatology 1985;5:1071-5.

21 Ikeda T, Pignatelli M, Lever AM, Thomas HC. Relationship of HLA protein display to activation of $2-5 \mathrm{~A}$ synthtase in $\mathrm{HBe}$ antigen or anti-BHe antigen positive chronic HBV infection. Gut 1986;27:1498-1501.

22 Nagafuchi Y, Scheuer PJ. Expression of $\beta_{2}$-microglobulin on hepatocytes in acute and chronic type $B$ hepatitis. Hepatology 1986;6:20-3.

23 Nagafuchi Y, Scheuer PJ. Hepatic $\beta_{2}$-microglobulin distribution in primary biliary cirrhosis. J Hepatol 1986; 2:73-80.

24 Pignatelli $M$, Waters J, Brown D, et al. HLA class I antigens on the hepatocyte membrane during recovery from acute hepatitis $B$ virus infection and during interferon therapy in chronic hepatitis B virus infection. Hepatology 1986;6:349-53.

25 Van De Oord JJ, De Vos R, Desmet V. In situ distribution of major histocompatibility complex products and viral antigens in chronic hepatitis $B$ virus infection: evidence that $\mathrm{HBC}$-containing hepatocytes may express HLA-DR antigens. Hepatology 1986;6:981-8.

26 Ballardini G, Bianchi FB, Mirikian R, et al. HLA-A, B, C HLA-D/DR and HLA-D/DQ expression on unfixed liver biopsy sections from patients with chronic liver disease. Clin Exp Immunol 1987;70:35-46.

27 Hubscher SG, Adams DH, Elias E. $\beta_{2}$-microglobulin expression in the liver after liver transplantation. $J$ Clin Pathol 1988;41:1049-57.

28 Chu CM, Shyu WC, Kuo RW, Liaw YF. HLA class I antigen display on hepatocyte membrane in chronic antigen display on hepatocyte membrane in chronic hepatitis B virus infection: its role in the pathogenes
chronic type B hepatitis. Hepatology 1988;8:712-17.

29 Brodsky FM, Parham P. Monomorphic anti-HLA-A, B, C monoclonal antibodies detecting molecular subunits and combinatorial determinants. J Immunol 1982;128:129-35.

30 Lampson LA, Levy R. Two populations of Ia-like molecule on a human B cell line. J Immunol 1980;125:293-9.

31 Ishii N, Klei J, Nagy Z. Different repertoires of mouse T cells for bovine insulin presented by syngeneic and allogeneic cells. Eur J Immunol 1983;13:658-62.

32 Mieli-Vergani G. Lymphocyte cytotoxicity to autologous hepatocytes in patients with chronic active hepatitis. $(\mathrm{PhD}$ thesis), University of London, 1985:88-104.

33 Gerken G, Manns M, Ramadori G, Poralla T, Dienes HP, Meyer zum Büschenfelde KH. Liver membrane autoantibodies in chronic active hepatitis. Studies on mechanically and enzymaticall 